\title{
Obtention of Antimicrobial Fibers Type Core/Shell PLA/PVOH-LAE by Coaxial Electrospinning
}

\section{Obtención de Fibras Antimicrobianas Tipo Pared/Núcleo PLA/PVOH-LAE Mediante Electrohilado Coaxial}

\author{
C. Patiño Vidal ${ }^{1,2}$, E. Velásquez ${ }^{1,2}$, M.J. Galotto ${ }^{1,2}$, and C. López de Dicastillo ${ }^{1,2}$
}

VII International Congress of

Science, Technology,

Entrepreneurship and

Innovation (SECTEI 2020)

Corresponding Author:

C. Patiño Vidal

cristian.patino@usach.c

Published: 26 August 2021

Production and Hosting by

Knowledge $E$

(c) C. Patiño Vidal et al. This article is distributed under the terms of the Creative Commons Attribution License, which permits unrestricted use and redistribution provided that the original author and source are credited.
${ }^{1}$ Centro de Innovación en Envases y Embalajes (LABEN-Chile), Departamento de Ciencia y Tecnología de los Alimentos, Facultad Tecnológica, Universidad de Santiago de Chile (USACH), Chile

${ }^{2}$ Centro para el Desarrollo de la Nanociencia y Nanotecnología (CEDENNA), Universidad de Santiago de Chile, Chile

\section{Abstract}

Coaxial electrospinning (EC) is a technology that allows the encapsulation of active compounds, as ethyl lauroyl arginate (LAE), in shell/core structures, in order to develop new antimicrobial food packaging materials with slowdown of active compound's release with the purpose of extending food shelf life. For this reason, the objective of this study consisted in developing antimicrobial fibers shell/core type by EC. Two polymers with different hydrophilic character as poly (lactic acid) (PLA) to the shell and poly (vinyl alcohol) (PVOH) and LAE to the core were used to obtain PLA/PVOH-LAE fibers, and slowdown the release of antimicrobial compound. The morphology of fibers was evaluated by optical microscopy and the thermal properties through thermogravimetric analyses (TGA). Release studies were carried out in fatty (ETOH 95\%) food simulant and was compared with the minimum inhibitory concentration (MIC) values of LAE against Listeria innnocua. The optical micrographs evidenced the obtention of shell/core structure with an average diameter of 0,6 $\mu \mathrm{m}$ approximately, and the TGA analyses demonstrated the thermal protection of LAE by shell of fibers. Released LAE reached the equilibrium state in $\mathrm{ETOH}$ 95\% during the first 3 hours, maintaining a higher concentration than MIC value obtained in $\mathrm{L}$. innnocua $(10 \mathrm{ppm})$. The results demonstrate that new packaging materials with antimicrobial activity as PLA/PVOH-LAE polymeric fibers with shell/core structure can be obtained through coaxial electrospinning technique.

Keywords: coaxial electrospinning, ethyl lauroyl arginate, fibers, shell/core, food packaging.

\section{Resumen}

El electrohilado coaxial (EC) es una tecnología que permite encapsular compuestos activos como el etil lauroil arginato (LAE) en estructuras tipo pared/núcleo con la finalidad de desarrollar nuevos materiales antimicrobianos para el envasado de alimentos que ralenticen la liberación del compuesto y extiendan la vida útil del alimento. Por tal razón, el objetivo de este estudio consistió en desarrollar fibras antimicrobianas tipo pared/núcleo mediante EC. Dos polímeros con distinto carácter hidrofílico como el poli (ácido láctico) (PLA) para la pared y poli (alcohol vinílico) (PVOH) y LAE para el núcleo se utilizaron para obtener las fibras PLA/PVOH-LAE, y ralentizar la liberación del compuesto antimicrobiano. La morfología de las fibras se evaluó mediante microscopía óptica y sus propiedades térmicas mediante análisis termogravimétricos 
(TGA). Se realizaron estudios de liberación del LAE en un simulante de alimentos graso (ETOH 95\%), y se comparó con la concentración mínima inhibitoria (MIC) hacia una bacteria Gram positiva Listeria innnocua. Las micrografías ópticas evidenciaron la obtención de la estructura pared/núcleo con un diámetro promedio de 0,6 $\mu \mathrm{m}$ aproximadamente, y el análisis TGA demostró la protección térmica del LAE por la pared de las fibras. El LAE liberado alcanzó el estado de equilibrio en ETOH 95\% durante las 3 hr iniciales, manteniendo una concentración superior a la MIC obtenida para L. innnocua (10 ppm). Los resultados demuestran que nuevos materiales de envase con actividad antimicrobiana como fibras poliméricas PLA/PVOH-LAE con estructura pared/núcleo pueden ser obtenidos mediante la técnica de electrohilado coaxial.

Palabras Clave: electrohilado coaxial, etil lauroil arginato, fibras, pared/núcleo, envase de alimentos.

\section{Introducción}

Actualmente, la nanotecnología es una importante herramienta que permite el uso de novedosas técnicas como el electrohilado para el desarrollo de nuevos materiales de envases activos para alimentos. El electrohilado es una técnica simple, eficiente, rentable con la capacidad de generar estructuras fibrilares a partir de una disolución polimérica [1]. Las fibras son obtenidas cuando un voltaje se aplica a la disolución polimérica, obteniendo un chorro con una estructura cónica conocida como 'Cono de Taylor', donde el solvente se evapora durante el proceso para finalmente obtener estructuras ultrafinas con una alta relación superficie/volumen [2].

Diversos polímeros pueden utilizarse para obtener estructuras fibrilares, como el poli(ácido láctico) (PLA) y el poli(alcohol vinílico) (PVOH). EI PLA es un polímero termoplástico alifático lineal proveniente de la fermentación del maíz, papa o caña, y, de acuerdo con la Administración de Alimentos y Drogas (FDA), los materiales de envase basados en este polímero son generalmente reconocidos como seguros (GRAS) [3, 4]. El PVOH es un polímero sintético, hidrofílico, semicristalino, biocompatible, biodegradable, no tóxico, barato, procesable y ampliamente utilizado en la industria [5]. Además, con el electrohilado es posible funcionalizar estos biopolímeros mediante la incorporación de agentes activos. Algunos compuestos activos como aceites esenciales, antioxidantes y antimicrobianos pueden utilizarse para brindarle actividad al material de envase, con la finalidad de extender la vida útil de los alimentos. Un compuesto con una elevada actividad antibacteriana es el etil lauroil arginato (LAE), surfactante catiónico derivado de sustancias naturales como el ácido láurico, L-arginina y etanol. Esta sustancia es un polvo higroscópico blanco, considerado GRAS por la FDA y autorizado como conservante alimentario por la Autoridad Europea de Seguridad Alimentaria (EFSA) [6]. 
Sin embargo, la rápida liberación que puede presentar la incorporación de este compuesto en este tipo de estructuras conlleva al uso de técnicas más innovadoras como el electrohilado coaxial (EC). Esta novedosa tecnología permite obtener estructuras tipo pared/núcleo con la finalidad de ralentizar la liberación de las sustancias activas incorporadas. Mediante este sistema se ha estudiado la liberación de antibióticos en el área de farmacéutica [7], en biomedicina para la ingeniería de tejidos [8] y en la industria de alimentos para la conservación de productos [9]. De esta forma, el desarrollo de fibras antimicrobianas tipo pared/núcleo mediante EC se postula como una manera novedosa de generar nuevos materiales de envases de alimentos con la capacidad de extender la vida útil de diferentes tipos de alimentos perecibles (carnes, embutidos, etc.).

Por esa razón, el objetivo del presente trabajo de investigación fue desarrollar fibras antimicrobianas tipo pared/núcleo mediante la técnica de electrohilado coaxial, donde la pared de las estructuras está compuesta por PLA mientras que el núcleo por PVOH y el compuesto antimicrobiano LAE.

\section{Metodología}

\subsection{Materiales}

\subsubsection{Reactivos químicos y microorganismos}

Poli(ácido láctico) 2003D se obtuvo de Natureworks ${ }^{\circledR}$ (Estados Unidos), poli(alcohol vinílico) Gohsenol tipo AH-17 se obtuvo de The Nippon Synthetic Chemical Co. (Japón), y etil lauroil arginato fue suministrado por PRINAL (Chile). Una bacteria Gram positiva de la orden de los Bacillales y familia Listeriaceae como la bacteria Listeria innocua ATCC 33090 se obtuvo del Laboratorio de Biotecnología y Microbiología Aplicada (LAMAP) (Chile). Cloroformo (CLF), dimetilformamida (DMF) y etanol (ETOH) se obtuvieron de Sigma Aldrich (Chile).

\subsection{Metodología}

\subsubsection{Actividad antimicrobiana del LAE}

La actividad antibacteriana del LAE se evaluó frente a una bacteria Gram+ (L. innocua) mediante la determinación de la concentración mínima inhibitoria (MIC) y concentración mínima bactericida (MBC). 100 uL de una concentración celular de 105 UFC/mL de la bacteria obtenidos por dilución se colocaron en tubos con $10 \mathrm{~mL}$ de caldo nutritivo. Diferentes concentraciones de LAE se colocaron en los tubos y se incubaron a $37^{\circ} \mathrm{C}$ y 
150 rpm durante 24 hr. Posteriormente, se realizaron diluciones seriadas y siembra en placa para determinar las concentraciones celulares obtenidas tras el contacto con el compuesto antimicrobiano. El diseño experimental utilizado para la determinación de la actividad antibacteriana del LAE abarcó el análisis de un factor que fue la concentración expresada en partes por millón (ppm) con siete niveles los cuales fueron: 10, 20, 30, 40, 50, 60, 70 ppm.

\subsection{Desarrollo}

\subsubsection{De fibras antimicrobianas PLA/PVOH-LAE}

Las fibras antimicrobianas tipo pared/núcleo se obtuvieron mediante un sistema de electrohilado coaxial (Spraybase ${ }^{\circledR}$ Power Supply Unit, Irlanda). La pared de las fibras se obtuvo a partir del proceso de electrohilado de una disolución de PLA al 10\% (p/v) en cloroformo y dimetilformamida en relación CLF/DMF (7:3), mientras que el núcleo con una disolución acuosa de PVOH al 10\% (p/v) y LAE al 2,5\% (p/p).

Las dos disoluciones se colocaron en jeringas de $5 \mathrm{~mL}$ y se llevaron al EC para obtener las fibras coaxiales PLA/PVOH-LAE con un flujo externo de $1,8 \mathrm{~mL} / \mathrm{h}$, un flujo interno de $0,5 \mathrm{~mL} / \mathrm{h}$ y una distancia de $14 \mathrm{~cm}$. Además, fibras control de PLA, PVOH y $P V O H$ con LAE se obtuvieron con la finalidad de evaluar y comparar sus propiedades térmicas frente a las fibras antimicrobianas PLA/PVOH-LAE.

\subsection{Caracterización de las fibras antimicrobianas}

\subsubsection{Microscopía óptica}

La morfología de las fibras PLA/PVOH-LAE se observó con un microscopio óptico (Zeiss Standard 25 ICS, Alemania) con una magnificación de 40x.

\subsubsection{Análisis termogravimétricos (TGA)}

Análisis TGA se realizaron mediante un sistema Stare System TGA/DSC (Mettler Toledo GC20, Suiza) con la finalidad de evaluar la estabilidad térmica de las fibras y protección del LAE en las estructuras. 5 a 6 mg de muestra se colocaron en cápsulas de alúmina y se calentaron de $30^{\circ} \mathrm{C}$ a $600^{\circ} \mathrm{C}$, con una velocidad de calentamiento de $10^{\circ} \mathrm{C} / \mathrm{min}$ bajo una atmósfera de nitrógeno (N2). 


\subsection{Estudio de liberación}

La liberación del LAE desde las fibras antimicrobianas se evaluó en un simulante de alimentos graso (ETOH 95\%) de acuerdo con el Reglamento No. 10/2011 de la Unión Europea sobre materiales y objetos plásticos destinados a entrar en contacto con alimentos.

El material se puso en contacto con el simulante en una relación área/volumen de 6 $\mathrm{dm}^{2} / \mathrm{L}$ a $40^{\circ} \mathrm{C}$, y periódicamente el LAE liberado se analizó mediante un cromatógrafo líquido de alta eficiencia (HPLC) (Waters Alliance, EEUU). $20 \mu \mathrm{L}$ de cada muestra se inyectaron en el HPLC equipado con una columna cromatográfica de fase inversa Waters Symmetry ${ }^{\circledR} \mathrm{C} 18(5 \mu \mathrm{m}, 3,9 \times 150 \mathrm{~mm})$, utilizando un flujo de $1 \mathrm{~mL} / \mathrm{min}$ de una mezcla acetonitrilo-agua (50:50) acidificada con 0,1\% de ácido trifluoracético como fase móvil.

\subsection{Análisis estadístico}

El análisis estadístico de la actividad antimicrobiana del LAE se realizó mediante un análisis de varianza (ANOVA de una vía) para detectar las diferencias entre las muestras $(p<0,05)$ con un nivel de confianza del 95\%, y posteriormente se realizó una comparación de muestras a través de la prueba de Tukey, utilizando el programa estadístico InfoStat (2016).

\section{Resultados y Discusión}

\subsection{Actividad antimicrobiana del LAE}

Los resultados de la MIC y MBC del compuesto antimicrobiano LAE están expresados en la Tabla 1. Se observó la elevada actividad antibacteriana del compuesto obteniendo una MIC de $10 \mathrm{ppm}$ para L. innocua. Al comparar la MIC de este aditivo alimentario frente a la MIC de compuestos sintéticos, aceites esenciales o nanopartículas, se evidencia su excelente y eficaz actividad antimicrobiana a concentraciones muy bajas $[10,11]$. Además, se observó que no existieron diferencias significativas de la actividad antibacteriana del LAE cuando concentraciones entre 20 y 60 ppm se utilizaron.

Por otro lado, se pudo evidenciar que una concentración de 70 ppm indujo un efecto bactericida en la $L$. innocua. Resultados similares fueron obtenidos por Higueras y col. (2013), los cuales obtuvieron una MIC de 8 ppm en L. monocytogenes [12]. La actividad antibacteriana del LAE se atribuye a su comportamiento como surfactante catiónico, ya que debido a su estructura química es capaz de reaccionar con la membrana de las bacterias. 
Esto a su vez produce un incremento de la permeabilidad entre la membrana celular y el citoplasma en las bacterias Gram+, produciendo la inhibición de los procesos metabólicos celulares [13]. Además, al comparar los resultados obtenidos en esta investigación frente a valores obtenidos en otros microorganismos que causan el deterioro de alimentos, se observa una elevada actividad antibacteriana del compuesto frente a $L$. innocua.

Por ejemplo, valores MIC mayores a 25 ppm han sido obtenidos en bacterias Gram negativas como la Escherichia coli o Salmonella enterica [6]. Esto se debe principalmente a que estas bacterias presentan una membrana externa que rodea la pared celular, la cual restringe la difusión de compuestos hidrófobos a través de su cubierta de lipopolisacáridos [14].

\section{Table 1}

Determinación de la MIC y MBC del LAE.

\begin{tabular}{lll} 
Concentración $(\mathbf{p p m})$ & UFC/mL & Reducción logarítmica \\
\hline 0 & $(1,14 \pm 0,01) \times 109$ & 0 \\
10 & $(5,68 \pm 0,75) \times 104$ & $4,76 \pm 1,05^{a}$ \\
20 & $(1,80 \pm 1,27) \times 103$ & $5,86 \pm 0,34^{b}$ \\
30 & $(3,33 \pm 0,33) \times 103$ & $5,69 \pm 0,55^{b}$ \\
40 & $(1,94 \pm 0,26) \times 103$ & $6,27 \pm 1,12^{b}$ \\
50 & $(2,61 \pm 0,00) \times 103$ & $5,64 \pm 0,10^{b}$ \\
60 & $(1,69 \pm 0,00) \times 103$ & $5,83 \pm 0,25^{b}$ \\
70 & 0 & $9,06 \pm 0,15^{c}$ \\
\hline$a-c$ Diferencias significativas entre las muestras $(p<0,05)$ de acuerdo al análisis de varianza y test de \\
Tukey.
\end{tabular}

\subsection{Propiedades morfológicas}

La Figura 1 muestra la morfología de las fibras antimicrobianas PLA/PVOH- LAE obtenidas mediante electrohilado coaxial. Las condiciones utilizadas durante el proceso electrodinámico permitieron obtener fibras uniformes y gruesas, con una estructura coaxial formada por una pared de PLA y un núcleo de PVOH y LAE (Figuras 1A y 1B). A pesar de que la técnica utilizada para observar las fibras no es la más apropiada para medir su diámetro, se determinó un tamaño promedio de 0,60 $\mu \mathrm{m}$ aproximadamente. A diferencia de otros resultados donde estructuras poliméricas pared/núcleo tuvieron diámetros entre 170 y 250 nm [15], las fibras coaxiales PLA/PVOH-LAE obtenidas en este trabajo tuvieron un diámetro mayor.

Se muestra las curvas TGA de la pérdida de masa respecto a la temperatura y sus correspondientes derivadas del compuesto antimicrobiano LAE, las fibras de PLA y $\mathrm{PVOH}$, así como de la estructura pared/núcleo PLA/PVOH- LAE. Las fibras de PLA 
comenzaron a degradarse térmicamente a los $280^{\circ} \mathrm{C}$ con una temperatura máxima de degradación de $353^{\circ} \mathrm{C}$ atribuido a la ruptura de la cadena polimérica, mientras que las fibras de $\mathrm{PVOH}$ evidenciaron dos procesos de degradación a $322^{\circ} \mathrm{C}$ y $433^{\circ} \mathrm{C}$, asociados a la separación de grupos laterales y degradación de la cadena principal del polímero $[16,17]$.

El compuesto antimicrobiano LAE evidenció un proceso de degradación entre los $200^{\circ} \mathrm{C}$ y $450^{\circ} \mathrm{C}$, resultado similar reportado en trabajos anteriores [18]. La incorporación del LAE en las fibras de PVOH produjo una reducción de la estabilidad térmica del polímero, lo cual puede estar atribuido a las posibles interacciones ocurridas entre el polímero y el surfactante.

Resultados similares han sido reportados cuando se ha incorporado LAE en polietileno de baja de densidad y quitosano $[18,19]$. Por otro lado, la obtención de las fibras antimicrobianas PLA/PVOH-LAE mostraron una reducción de la estabilidad térmica del PLA con una temperatura máxima de degradación de $330^{\circ} \mathrm{C}$, asociado probablemente a la degradación temprana del PVOH [20].

\subsection{Estudio de liberación}

Se muestra la cinética de liberación del LAE en el simulante de alimentos graso a $40^{\circ} \mathrm{C}$. El estudio se realizó durante $72 \mathrm{hr}$ con la finalidad de observar el comportamiento de la liberación del compuesto durante un tiempo prolongado y obtener el estado de equilibrio. De acuerdo con la Figura 2A, la liberación del LAE a partir de las fibras PLA/PVOH-LAE mostraron un comportamiento exponencial, logrando el equilibrio a partir de las 3 primeras horas. Una concentración máxima de 14 ppm evidenció que el 98\% del LAE presente en la estructura pared/núcleo se liberó en el simulante graso.

Esto se explica por la interacción plastificante que presenta el PLA con el etanol, provocando un hinchamiento de la matriz polimérica, la modificación de sus propiedades de transporte y como consecuencia una alta liberación del compuesto activo [21]. Además, una rápida liberación del LAE se pudo evidenciar en el simulante durante la primera hora de estudio (Figura 2B), relacionado con el rápido efecto plastificante que ocasiona el agua y etanol en el PVOH. Esto es debido a que la molécula de agua es más pequeña que la molécula de LAE, por lo que su ingreso en la matriz es tan rápida que logra equilibrar la liberación del compuesto activo en las primeras etapas [12, 22].

Al comparar el resultado obtenido de la liberación del LAE respecto a su actividad antibacteriana, se evidencia que es posible superar la MIC de la L. innocua al exponerlo a un ambiente graso a partir de las $3 \mathrm{hr}$. Por lo cual, si este material se utilizara para ponerse en contacto con algún alimento rápidamente perecible por la presencia de esta bacteria, seguramente permitiría mantener y extender su vida útil. 


\section{Conclusiones}

En este estudio se obtuvieron satisfactoriamente fibras antimicrobianas con una estructura pared/núcleo y un diámetro aproximado de 0,6 $\mu \mathrm{m}$ mediante electrohilado coaxial. La pared de la estructura coaxial protegió térmicamente al compuesto antimicrobiano $\mathrm{LAE}$, pero su incorporación produjo una reducción de la estabilidad térmica del PVOH al igual que la del PLA cuando se obtuvo la estructura coaxial PLA/PVOH-LAE. Se observó una rápida liberación del LAE en el simulante de alimentos graso, alcanzando el equilibrio a partir de las $3 \mathrm{hr}$ con una concentración superior (14 ppm) que la MIC obtenida para L. innocua.

\section{Agradecimientos}

Los autores agradecen a la Beca de Doctorado CONICYT-PFCHA/Doctorado Nacional/ 2019-21190316 y al proyecto FONDECYT REGULAR 1200766.

\section{Conflicto de Intereses}

Los autores declaran no tener conflictos de intereses.

\section{References}

[1] Ali A, Gómez-Mascaraque L, Marta M-S, López-Rubio A. Electrospun curcuminloaded protein nanofiber mats as active/bioactive coatings for food packaging applications. Food Hydrocoll. 2019;87:758-71.

[2] Quiles-Carrillo L, Montanes N, Lagaron J, Balart R, Torres-Giner S, Quiles-Carrillo L. Bioactive multilayer polylactide films with controlled release capacity of gallic acid accomplished by incorporating electrospun nanostructured coatings and interlayers. Appl Sci. 2019;9(3):533.

[3] Armentano I, Bitinis N, Fortunati E et al. Multifunctional nanostructured PLA materials for packaging and tissue engineering. Prog Polym Sci. 2013;38(10-11):1720-47.

[4] Lima EMB, Lima AM, Minguita APS et al. Poly(lactic acid) biocomposites with mango waste and organo-montmorillonite for packaging. J Appl Polym Sci. 2019;136(21):47512.

[5] Wang B, Chen Z, Zhang J et al. Fabrication of PVA/graphene oxide/TiO2 composite nanofibers through electrospinning and interface sol-gel reaction: Effect of graphene oxide on PVA nanofibers and growth of TiO2. Colloids Surfaces A Physicochem Eng Asp. 2014;457:318-25. 
[6] Nerin C, Becerril R, Manso S, Silva F. Ethyl lauroyl arginate (LAE): Antimicrobial activity and applications in food systems. Antimicrob Food Packag. 2016;305-12.

[7] Zupančič Š. Core-shell nanofibers as drug delivery systems. Acta Pharm. 2019;69(2):131-53.

[8] Johnson R, Ding Y, Nagiah N, Monnet E, Tan W. Coaxially-structured fibres with tailored material properties for vascular graft implant. Mater Sci Eng C. 2019;97:1-11.

[9] Yao Z-C, Chang M-W, Ahmad Z, Li J-S. Encapsulation of rose hip seed oil into fibrous zein films for ambient and on demand food preservation via coaxial electrospinning. J Food Eng. 2016;191:115-23.

[10] Hajipour M, Fromm K, Ashkarran A et al. Antibacterial properties of nanoparticles. Trends Biotechnol. 2012;30(10):499-511.

[11] Van de Vel E, Sampers I, Raes K. A review on influencing factors on the minimum inhibitory concentration of essential oils. Critical Reviews in Food Science and Nutrition. 2019;59:357-78.

[12] Higueras L, López-Carballo G, Hernández-Muñoz P, Gavara R, Rollini M. Development of a novel antimicrobial film based on chitosan with LAE (ethyl-N $\alpha$ - dodecanoyl-Iarginate) and its application to fresh chicken. Int J Food Microbiol. 2013;165(3):33945.

[13] Haghighi H, De Leo R, Bedin E, Pfeifer F, Siesler HW, Pulvirenti A. Comparative analysis of blend and bilayer films based on chitosan and gelatin enriched with LAE (lauroyl arginate ethyl) with antimicrobial activity for food packaging applications. Food Packag Shelf Life. 2019;19:31-9.

[14] Muriel-Galet V, López-Carballo G, Gavara R, Hernández-Muñoz P. Antimicrobial food packaging film based on the release of LAE from EVOH. Int J Food Microbiol. 2012;157(2):239-44. D

[15] da Silva TN, Gonçalves RP, Rocha CL et al. Controlling burst effect with PLA/PVA coaxial electrospun scaffolds loaded with BMP-2 for bone guided regeneration. Mater Sci Eng C. 2019;97:602-12.

[16] López de Dicastillo C, Patiño C, Galotto M, Palma J, Alburquenque D, Escrig J. Novel antimicrobial titanium dioxide nanotubes obtained through a combination of atomic layer deposition and electrospinning technologies. Nanomaterials. 2018;8(2):128.

[17] Gonçalves RP, da Silva FFF, Picciani PHS, Dias ML. Morphology and thermal properties of core-shell PVA/PLA ultrafine fibers produced by coaxial electrospinning. Mater Sci Appl. 2015;06(02):189-99.

[18] Gaikwad KK, Lee SM, Lee JS, Lee YS. Development of antimicrobial polyolefin films containing lauroyl arginate and their use in the packaging of strawberries. J Food Meas Charact. 2017;11(4):1706-16. 
[19] Deng L, Taxipalati M, Zhang A et al. Electrospun Chitosan/Poly(ethylene oxide)/Lauric arginate nanofibrous film with enhanced antimicrobial activity. J Agric Food Chem. 2018;66(24):6219-26.

[20] López de Dicastillo C, Garrido L, Alvarado N, Romero J, Palma J, Galotto M. Improvement of polylactide properties through cellulose nanocrystals embedded in poly(vinyl alcohol) electrospun nanofibers. Nanomaterials. 2017;7(5):106.

[21] Alvarado N, Romero J, Torres A et al. Supercritical impregnation of thymol in poly(lactic acid) filled with electrospun poly(vinyl alcohol)-cellulose nanocrystals nanofibers: Development an active food packaging material. J Food Eng. 2017;217:110.

[22] Muriel-Galet V, López-Carballo G, Hernández-Muñoz P, Gavara R. Characterization of ethylene-vinyl alcohol copolymer containing lauril arginate (LAE) as material for active antimicrobial food packaging. Food Packag Shelf Life. 2014;1(1):10-8. 